\title{
Genome-wide identification of ZmSnRK2 genes and functional analysis of ZmSnRK2.10 in ABA signaling pathway in maize (Zea mays L)
}

Tiandan Long ${ }^{1,2+}$, Binjie Xu ${ }^{3+}$, Yufeng Hu ${ }^{1,2}$, Yayun Wang ${ }^{2}$, Changqing Mao ${ }^{2}$, Yongbin Wang ${ }^{2}$, Junjie Zhang ${ }^{4}$,

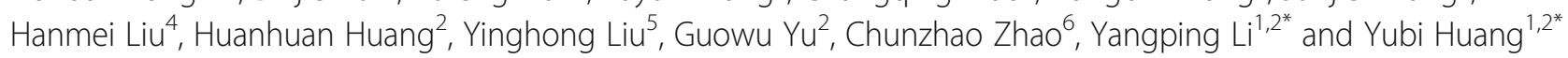

\begin{abstract}
Background: Phytohormone abscisic acid (ABA) is involved in the regulation of a wide range of biological processes. In Arabidopsis, it has been well-known that SnRK2s are the central components of the ABA signaling pathway that control the balance between plant growth and stress response, but the functions of ZmSnRK2 in maize are rarely reported. Therefore, the study of ZmSnRK2 is of great importance to understand the ABA signaling pathways in maize.

Results: In this study, 14 ZmSnRK2 genes were identified in the latest version of maize genome database. Phylogenetic analysis revealed that ZmSnRK2s are divided into three subclasses based on their diversity of Cterminal domains. The exon-intron structures, phylogenetic, synteny and collinearity analysis indicated that SnRK2s, especially the subclass III of SnRK2, are evolutionally conserved in maize, rice and Arabidopsis. Subcellular localization showed that ZmSnRK2 proteins are localized in the nucleus and cytoplasm. The RNA-Seq datasets and qRT-PCR analysis showed that ZmSnRK2 genes exhibit spatial and temporal expression patterns during the growth and development of different maize tissues, and the transcript levels of some ZmSnRK2 genes in kernel are significantly induced by ABA and sucrose treatment. In addition, we found that ZmSnRK2.10, which belongs to subclass III, is highly expressed in kernel and activated by ABA. Overexpression of ZmSnRK2.10 partially rescued the ABA-insensitive phenotype of snrk2.2/2.3 double and snrk2.2/2.3/2.6 triple mutants and led to delaying plant flowering in Arabidopsis.
\end{abstract}

Conclusion: The SnRK2 gene family exhibits a high evolutionary conservation and has expanded with wholegenome duplication events in plants. The ZmSnRK2s expanded in maize with whole-genome and segmental duplication, not tandem duplication. The expression pattern analysis of ZmSnRK2s in maize offers important information to study their functions. Study of the functions of ZmSnRK.10 in Arabidopsis suggests that the ABAdependent members of SnRK2s are evolutionarily conserved in plants. Our study elucidated the structure and evolution of SnRK2 genes in plants and provided a basis for the functional study of ZmSnRK2s protein in maize.

Keywords: Maize, ABA, SnRK2 family, Evolution, Expression pattern, ZmSnRK2.10, Function analysis

\footnotetext{
* Correspondence: ypli@sicau.edu.cn; yubihuang@sohu.com

${ }^{\dagger}$ Tiandan Long and Binjie Xu contributed equally to this work.

${ }^{1}$ State Key Laboratory of Crop Gene Exploration and Utilization in Southwest China, Chengdu 611130, Sichuan, China

Full list of author information is available at the end of the article
} 


\section{Background}

Phytohormone abscisic acid (ABA) is widely involved in plant growth and development, responses to biotic and abiotic stresses, as well as crop grain filling and seed maturation processes $[1,2]$. The core regulatory components of ABA signaling have been identified and studied well in Arabidopsis. ABA binds to the receptors pyrabactin resistance 1 (PYR1) and PYR1-like proteins (PYLs), which interact with and inhibit clade-A protein phosphatase type 2Cs (PP2Cs), leading to the release of SnRK2 (Sucrose non-fermenting protein kinase 2). The activated SnRK2s subsequently phosphorylate downstream target proteins such as ABA-responsive elementbinding factors (ABFs) to mediate various ABA responses [3-7]. SnRK2s are crucial for ABA signaling because ABA-mediated regulation of various physiological processes is attributed to the SnRK2s-mediated phosphorylation of different downstream targets.

The first $S n R K 2$ gene, named PKABA1, was cloned and characterized in wheat. PKABA1 is involved in the phosphorylation of downstream ABA responsive transcription factor TaABF1 [8]. So far, the SnRK2 proteins have been identified and characterized in many species, including 10 members (SnRK2.1 to - 2.10) in Arabidopsis [9] and 10 (SAPK1 to - 10) in rice [10]. SnRK2 family can be divided into three subclasses (subclass I, II and III) based on the sequence of amino acids at the Cterminal domain [9]. Analysis of the kinase activity of SnRK2s in Arabidopsis and rice indicates that each subclass of SnRK2 has a discrepant activation pattern in response to ABA and osmotic stress. Subclass I SnRK2s are quickly and strongly activated by osmotic stress, while the ABA-independent subclass II and III SnRK2s are activated by both ABA and osmotic stress. Specifically, subclass II SnRK2s are weakly activated by ABA, and subclass III SnRK2s are rapidly and strongly activated by ABA. Therefore, subclass III SnRK2s are widely considered as key regulators in ABA signaling pathway [10-14]. In Arabidopsis, subclass III SnRK2s are encoded by three genes, $S n R K 2.2$, $S n R K 2.3$, and $S n R K 2.6$ which are quickly activated by ABA treatment $[11,15]$. snrk2.2/2.3/2.6 triple mutant exhibits a small plant size and is insensitive to ABA in terms of seed germination and seeding growth [16]. Although SnRK2.2, SnRK2.3, and $S n R K 2.6$ function redundantly in ABA-mediated regulation of plant growth and stress responses, there also exhibit distinct functions in some aspects. SnRK2.6 is mainly required for $\mathrm{ABA}$-induced stomatal closure, and therefore snrk2.6 single mutant leads to severe water loss in Arabidopsis leaves [17, 18]. SnRK2.2 and SnRK2.3 are particularly involved in the regulation of seed germination and seedling growth. snrk2.2/2.3 double mutant can germinate in the presence of extremely high ABA concentration [19]. In rice, subclass III SnRK2s include three genes, SAPK8, SAPK9, and SAPK10 which are homologous to Arabidopsis $S n R K 2.2$, SnRK2.3, and SnRK2.6 respectively. These three SnRK2 proteins are also activated by ABA $[10,20]$. Compared with Wild type (WT), transgenic rice lines overexpressing $S A P K 8$, $S A P K 9$, or SAPK10 show delayed germination and seedling growth [21], suggesting that the subclass III SnRK2s in rice is also involved in the regulation of seeds germination.

Maize (Zea mays L.) not only serves as grain and forage crop but also is widely used as main raw material in many fields such as food and bioenergy industries. In maize, ABA coupled with the VIVIPAROUS1 (VP1) transcription factor plays an essential role in kernel development and maturation [22, 23]. In addition, ABA is also a key factor in the regulation of programmed cell death during maize endosperm development [24, 25]. Here, we conducted genome-wide identification of $Z m S n R K 2$ gene family in maize, and the gene structure, chromosomal location, phylogenetic relationship, synteny, and expression profiles of these $Z m S n R K 2 s$ were investigated. Moreover, we found that $Z m S n R K 2.10$ gene, which belongs to subclass III, was activated by ABA in maize. Overexpression of $Z m S n R K 2.10$ could partially complement the phenotype of Atsnrk2.2/2.3, Atsnrk2.2/2.3/2.6 mutants and delay the flowering time in Arabidopsis. This study will enhance our understanding of the $S n R K 2$ gene family and provide insights into the functional diversity of $S n R K 2$ genes in maize.

\section{Results}

Genome-wide identification and evolutionary analysis of SnRK2 genes in maize

To identify possible SnRK2 homologs in MaizeGDB database (https://maizegdb.org/), we constructed the HMM (Hidden Markov Model) of SnRK2s based on their protein sequences in Arabidopsis thaliana and Oryza sativa, and used it for BLASTP analysis. A total of 14 candidate $Z m S n R K 2$ genes were identified and characterized (Additional file 1: Table S1), among which 10 $Z m S n R K 2$ genes have been cloned and reported previously [26], we cloned the $Z n S n R K 2.9$ gene and the remaining three newly identified $Z m S n R K 2$ genes were designated ZmSnRK2.12 to ZmSnRK2.14. To characterize the properties of the identified SnRK2s in maize, the protein sequences of $\mathrm{ZmSnRK2s}$ were analyzed by ExPASy (https://web.expasy.org/compute_pi/). As shown in Table S1, the protein lengths of $\mathrm{ZmSnRK2s}$ range from 333 amino acids (ZmSnRK2.3) to 366 amino acids (ZmSnRK2.8 and ZmSnRK2.12) and the molecular weights range from $37.84 \mathrm{kDa}(\mathrm{ZmSnRK} 2.3)$ to 42.84 $\mathrm{kDa}(\mathrm{ZmSnRK} 2.5)$. The $\mathrm{ZmSnRK} 2$ proteins were acidic with predicted pI values varying from 4.57 to 6.73 . 
The full-length of $14 \mathrm{ZmSnRK} 2$ proteins downloaded from Gramene (http://ensembl.gramene.org/) were clustered by clustalw1.83, and then a neighbor-joining (NJ) phylogenetic tree was constructed (Additional file 1: Fig. S1A). Comparative analysis showed that $Z m S n R K 2 s$ were clustered into three subclasses, in which $Z m S n R K 2.12$ with $Z m S n R K 2.8, Z m S n R K 2.9$ and $Z m S n R K 2.10$ was classified as subclass III, while ZmSnRK2.13 and ZmSnRK2.14 with ZmSnRK2.4, ZmSnRK2.5, $Z m S n R K 2.6, Z m S n R K 2.7$ and $Z m S n R K 2.11$ were clustered into subclass I. Based on previous reports [26, 27], $Z m S n R K 2.3$ with $Z m S n R K 2.1$ and $Z m S n R K 2.2$ were classified as subclass II. The conserved motifs in ZmSnRK2 proteins were analyzed by MEME (http://meme-suite. org/), all the ZmSnRK2 proteins were identified to contain six large conserved motifs (Additional file 1: Fig. S1A). Among these proteins, the location of the conserved motifs in subclass III proteins showed a high similarity, while the other $10 \mathrm{ZmSnRK2s}$ (subclass I and subclass II) exhibited a similar pattern of motifs distribution. Protein sequences alignment indicated that the $\mathrm{ZmSnRK2s}$ were evolutionarily conserved, and the variations in $\mathrm{ZmSnRK} 2$ proteins were mainly identified at Cterminal regions (Additional file 1: Fig. S1B).

To investigate the evolutionary features of identified ZmSnRK2 genes in maize with that in other plants, phylogenetic and conserved motifs analysis were performed based on the amino acid sequences of all SnRK2 proteins from Zea mays, Arabidopsis thaliana and Oryza sativa. All these SnRK2s were clustered into three subclasses and contain six large conserved motifs too (Additional file 1: Fig. S2A, Fig. S2B). It is worth noting that $Z m S n R K 2.8 / 2.9 / 2.10 /$ 2.12 belong to the same branch with SnRK2.2/2.3/2.6 in Arabidopsis and SAPK8/9/10 in rice and exhibited a similar pattern of conserved motifs distribution (Fig. 1A). SnRK2.2/ 2.3/2.6 play a crucial role in responding to ABA signal pathway in Arabidopsis [28], which may suggest these four members of subclass III play an important function in response to ABA signal in maize. The other two newly identified $Z m S n R K 2$ genes ( $\mathrm{ZmSnRK2.13}$ and $\mathrm{ZmSnRK2.14)}$ were clustered with AtSnRK2s in Arabidopsis that are particularly involved in response to osmosis stress [16], which suggested a possible role of these two ZmSnRK2s in the regulation of osmotic stress in maize. The difference between subclass III with subclass I and subclass II was that aspartic acid (short for D) was enriched in the C-terminal regions of subclass III proteins, while glutamate (short for E) was enriched in the C-terminal regions of subclass I and II proteins (Fig. 1B and Additional file 1: Fig. S2C).

Genes structure, chromosomal localization and synteny analysis of ZmSnRK2 genes in Zea mays

To explore the structural diversity of the $Z m S n R K 2$ members, the intron-exon organization of each
$\mathrm{ZmSnRK2}$ gene was analyzed by comparing the cDNA sequences with the corresponding genomic DNA sequences. As show in Fig. 2A, most of $Z m S n R K 2$ genes contain nine exons and eight introns, whereas $Z m S n R K 2.5$ only consists of a single intron. Compared with other $Z m S n R K 2$ genes, ZmSnRK2.6 contains a long intron in its gene structure. Notably, three members (ZmSnRK2.1, ZmSnRK2.2 and ZmSnRK2.3) clustered in subclass I share a high similarity in gene structure. For four ZmSnRK2 members (ZmSnRK2.8, ZmSnRK2.9, $Z m S n R K 2.10$, and ZmSnRK2.12) clustered in subclass III, ZmSnRK2.8 and ZmSnRK2.12 share a high similarity in gene structure, as well as ZmSnRK2.9 and $Z m S n R K 2.10$. Besides, ZmSnRK2.14, ZmSnRK2.11 and $Z m S n R K 2.4$ share a high similarity in gene structure, as well as $Z m S n R K 2.13$ and $Z m S n R K 2.7$.

Combining the information of the highly conserved motifs, phylogenetic and gene structures analysis of $Z m S n R K 2 s$, we speculated that the expansion of ZmSnRK2s in maize tended to be tandem or segmental duplication events. Firstly, the physical locations of the $Z m S n R K 2$ genes on chromosomes were analyzed based on the genome database. As the Fig. 2B shown that all the identified ZmSnRK2 genes could be mapped on maize chromosomes from 1 to 10 , with each 3 on chromosome 1 and 5. Interestingly, four ZmSnRK2 members clustered in subclass III were located at the distal side of either chromosome 1 or chromosome 5 . Moreover, ZmSnRK2.8 and ZmSnRK2.9 are closely associated on chromosome 1 , while $Z m S n R K 2.10$ and $Z m S n R K 2.12$ are closely associated on chromosome 5 . In addition, we identified many segmental duplication events but not tandem duplication events among SnRK2s in maize by using MCScanX program. Furthermore, synteny and collinear relationships among Zea mays, Arabidopsis thaliana and Oryza sativa were analyzed to investigate potential evolutionary events of $S n R K 2 s$ in plants. Each member of SnRK2 in maize has one or more orthologous genes in Arabidopsis and rice, and this collinearity relationship occurs in the same subclass (Fig. 2C), suggesting that the duplication events of SnRK2s have occurred in plants before the differentiation of maize, rice, and Arabidopsis. Together, these results indicated that the expansion of SnRK2 genes family in maize mainly because of segmental duplication.

\section{Expression pattern analysis of ZmSnRK2 genes in Zea mays}

To explore the biological functions of $Z m S n R K 2$ genes, we first analyzed the expression profile of $Z m S n R K 2$ genes in 78 samples. These samples were derived from different tissues at different developmental stages of maize and the transcriptome data in these samples were downloaded from NCBI database (http://www.ncbi.nlm. 


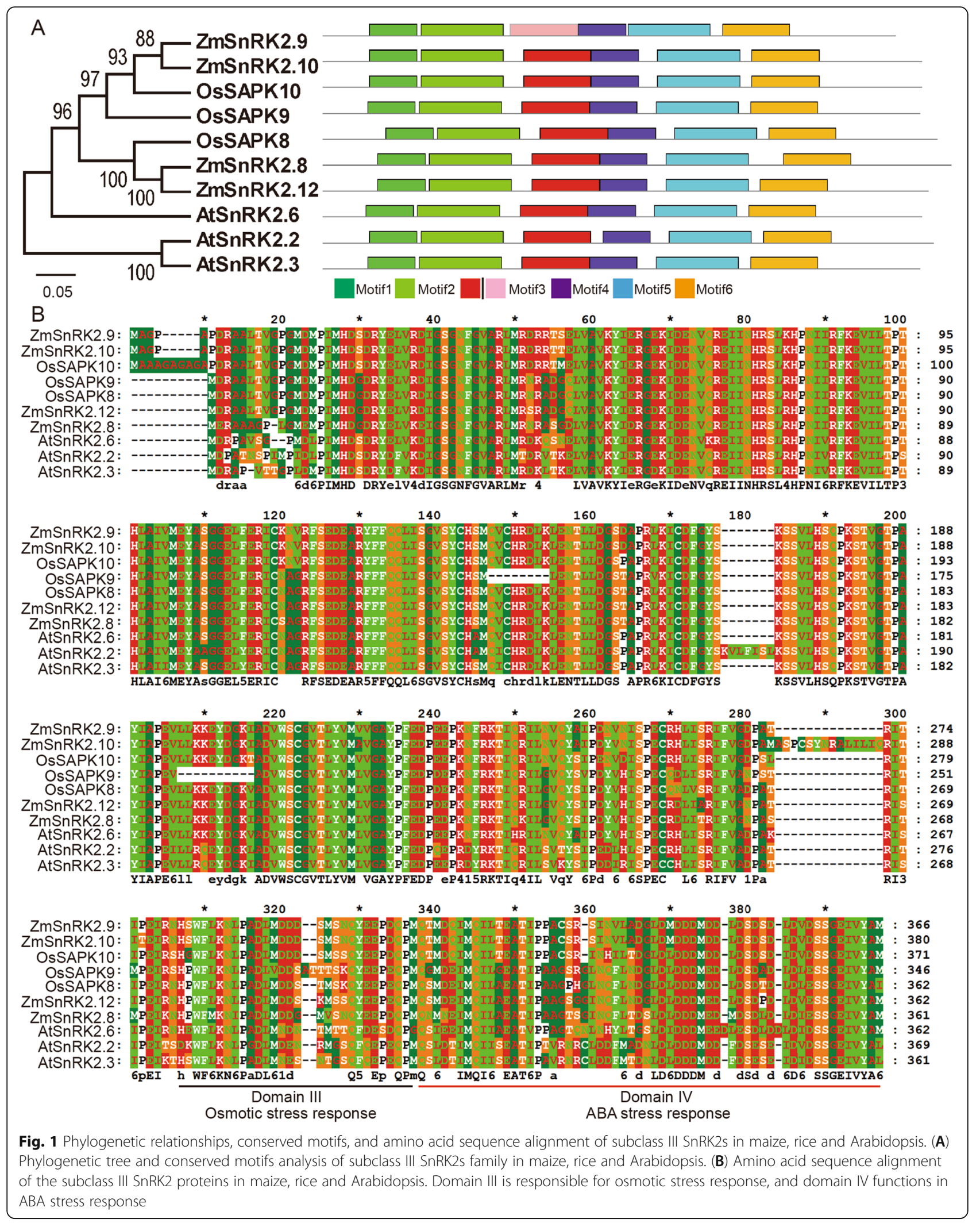




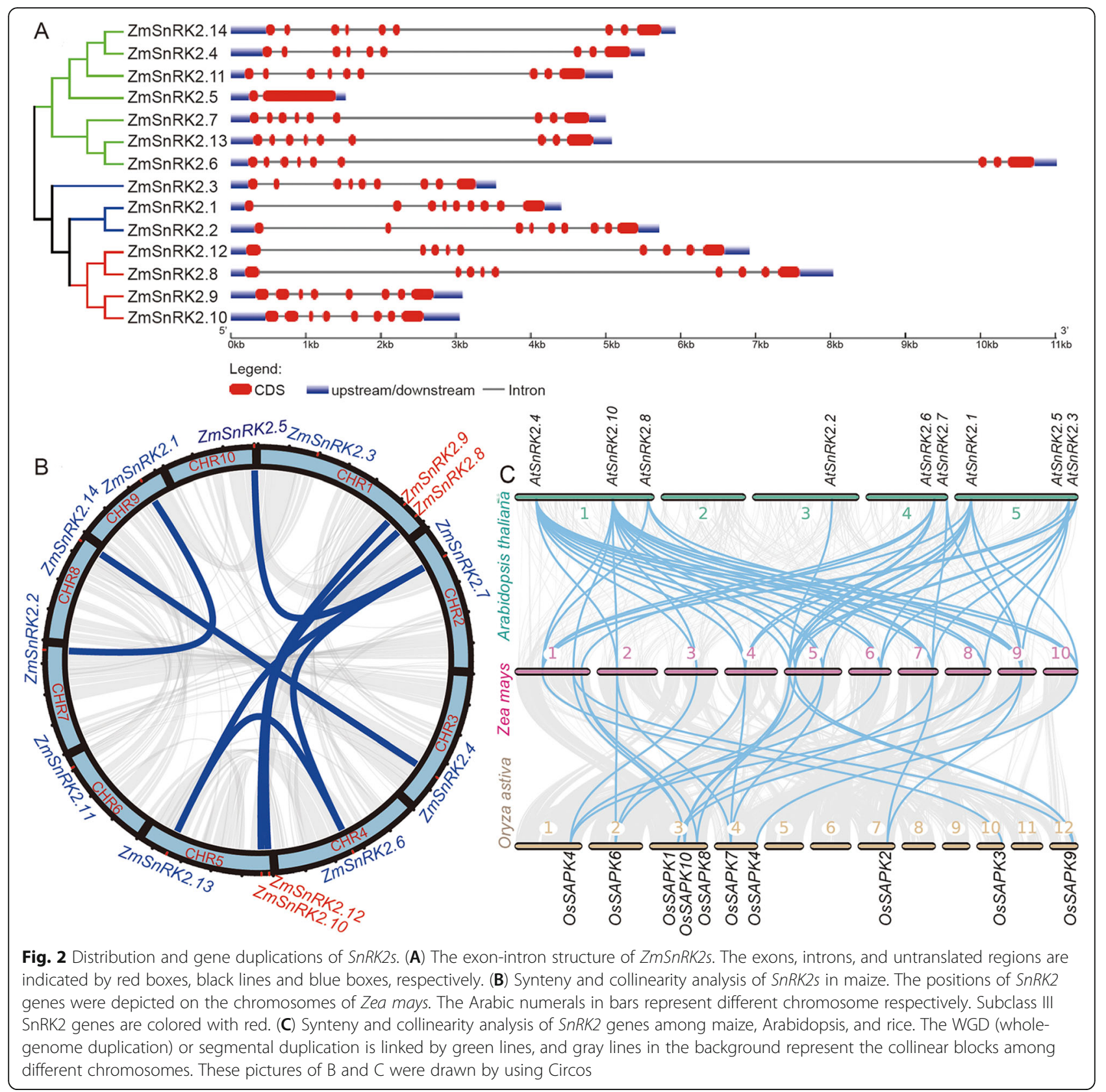

nih.gov/sra) [29]. The ZmSnRK2 genes showed different spatial and temporal expression patterns in maize (Fig. 3A). For the members of subclass III, the expression level of $Z m S n R K 2.8$ was relatively stable at each stage of the kernel development, but was significantly higher in the embryo than that of the endosperm. The expression level of $Z m S n R K 2.9$ was low in all samples. Notably, ZmSnRK2.10 was highly expressed during the development of kernel, especially in the 10 DAP (days after pollination) and 24 DAP of kernel development, the stage of which is very important for grain filling and seed maturation. ZmSnRK2.12 was the only gene that showed a high expression level in pollen. Besides, the expression level of $Z m S n R K 2.14$ was relative lower in almost all tissues, whereas the $Z m S n R K 2.13$ was highly expressed in seeds (Fig. 3A, B). The expression of ZmSnRK2 genes in several maize tissues or during kernel development was examined by semi-quantitative RT-PCR (sqRT-PCR) or qRT-PCR analysis, and results obtained from qRT-PCR analysis were almost consistent with those obtained from the public database (Figs. 3B4D). ABA and sucrose act as signal molecules to regulate the expression of genes involved in metabolism during grain filling $[2,30]$. We then analyzed the expression 


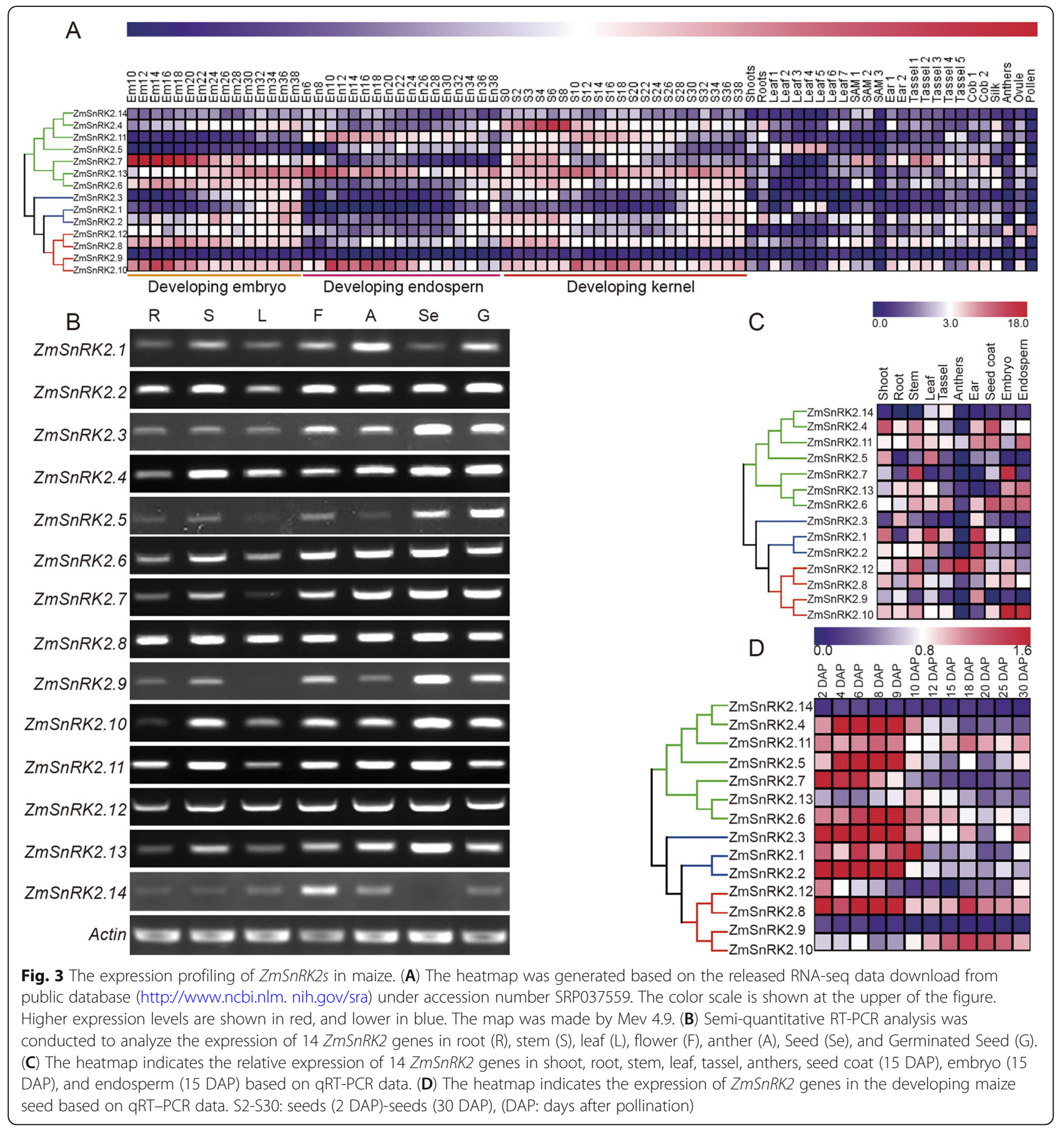

changes of the ZmSnRK2 genes in maize kernel under the treatment of $A B A$ and sucrose, and the result indicated that the ZmSnRK2 genes were differentially expressed after ABA or sucrose treatment (Additional file 1: Fig. S3). For example, the expression levels of $Z m S n R K 2.1 / 2.3 / 2.6 / 2.9 / 2.10$ were upregulated under ABA treatment, whereas the transcript levels of ZmSnRK2.8/2.9/2.10/1.12, members of subclass III, were increased after sucrose treatment. Besides, the expression level of $Z m S n R K 2.13$ was downregulated under both $\mathrm{ABA}$ and sucrose treatment.

ZmSnRK2 proteins are localized in cytoplasm and nucleus The cytoplasmic and nuclear localization of ZmSnRK2.1 to ZmSnRK2.11 (except ZmSnRK2.9) proteins in Nicotiana benthamiana leaves have been reported [31]. Here, to determine the subcellular localization of all $\mathrm{ZmSnRK2}$ proteins accurately, we generated $Z m S n R K 2 s-G F P$ fusion 


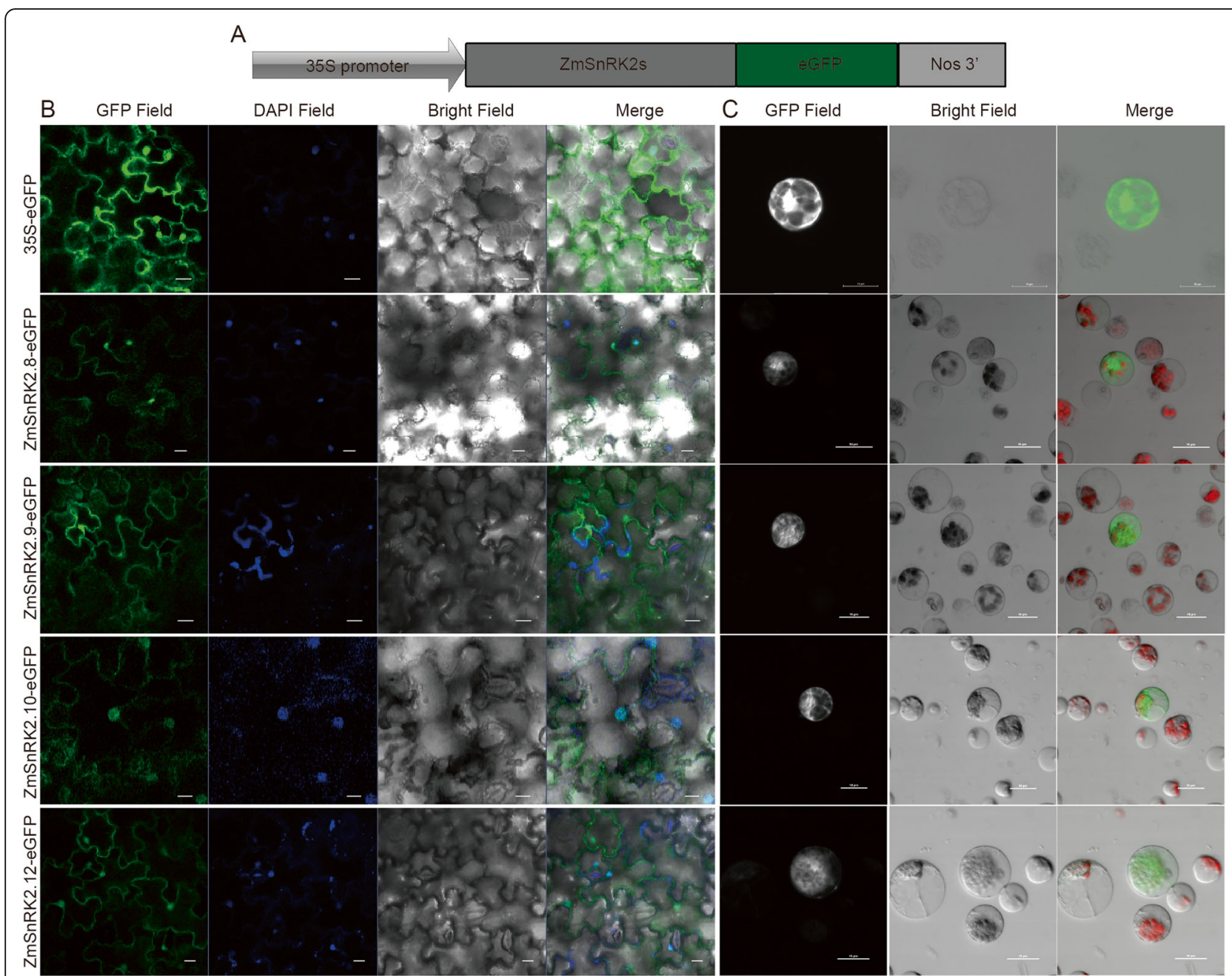

Fig. 4 Subcellular localization of subclass III SnRK2 proteins. (A) Schematic illustration of the constructs. (B) The construct containing ZmSnRK2s:GFP fusion protein vector into the Agrobacterium tumefaciens strain GV3101 and used to infect Nicotiana benthamiana leaf cell. The bars, $50 \mu \mathrm{m}$. (C) The ZmSnRK2s:GFP fusion protein was transformed into maize leaf protoplasts respectively. The bars, $10 \mu \mathrm{m}$. All fluorescence signals were detected using the Confocal microscope. 35S-eGFP was used as the control. Merged, individual images of GFP and bright field images of onion epidermal cells were shown. GFP, green fluorescent protein. The localization of the nuclei was indicated by DAPI (6-diamidino-2-phenylindole) staining

constructs driven by the cauliflower mosaic virus (CaMV) 35S promoter, and transiently transformed them to both maize protoplast and Nicotiana benthamiana leaves. A strong green fluorescence signal was observed in the nucleus and cytoplasm of the maize protoplasts or Nicotiana benthamiana leaves cells transformed with $Z m S n R K 2 s-G F P$, while the free GFP protein was detected in the cytoplasm, plasma membrane and nuclei (Fig. 4 and Additional file 1: Fig. S4).

\section{The kinase activity of ZmSnRK2.10 protein is activated by} ABA

Considering that the ZmSnRK2.10, a subclass III SnRK2s, was highly expressed during kernel development and was induced by ABA, we investigated whether the ZmSnRK2.10 protein kinases is activated by ABA and whether it is directly involved in $A B A$ signaling pathway. The luciferase reporter gene driven by the ABA-responsive $R D 29 B$ promoter was used to test the roles of $Z m S n R K 2.10$ in ABA signaling pathway. Four selected $Z m S n R K 2 s$ members, including $Z m S n R K 2.8$ ( $Z m O S T 1)$ that is activated by ABA and weakly mediates the closing of stomata in maize [32], ZmSnRK2.10, ZmSnRK2.11 (member of subclass I) and ZmSnRK2.3 (member of subclass II) were tested. The ZmSnRK2 genes and reporter gene were co-transformed into maize leaf protoplasts. The result showed that the protoplast expressing $Z m S n R K 2.8$ or $Z m S n R K 2.10$ exhibited a higher $R D 29 B$-LUC expression than that expressing ZmSnRK2.3 or ZmSnRK2.11. Similar to ZmSnRK2.8, the 
up-regulation of RD29B-LUC expression by $\mathrm{ZmSnRK2.10}$ was extremely enhanced by ABA treatment (Additional file 1: Fig. S5). To further confirm that the ZmSnRK2.10 protein kinase was directly activated by ABA, immunoprecipitation-kinase (IP-kinase) assay was performed using $\mathrm{MBP}$ as substrates to investigate the kinase activity of with or without ABA treatment. After immunoprecipitation of ZmSnRK2 proteins by anti-Flag antibody, in vitro kinase assay was performed. The result showed that none of these $\mathrm{ZmSnRK2s}$ exhibited kinase activity without ABA treatment. However, the kinase activities of $\mathrm{ZmSnRK} 2.8$ and $\mathrm{ZmSnRK2.10}$, but not $\mathrm{ZmSnRK2.3} \mathrm{and} \mathrm{ZmSnRK2.11,} \mathrm{were} \mathrm{induced} \mathrm{after} \mathrm{ABA}$ treatment (Fig. 5). These results indicated that both ZmSnRK2.8 and ZmSnRK2.10 are activated by ABA and are involved in the regulation of ABA-responsive genes expression.

\section{Overexpression of $Z m S n R K 2.10$ partially rescues the ABA- insensitive phenotype of snrk2.2/2.3 and snrk2.2/2.3/2.6 mutants}

To investigate the biological functions of $Z m S n R K 2 s$, we generated Arabidopsis transgenic plants expressing four selected $Z m S n R K 2$ genes $(Z m S n R K 2.3 / 2.8 / 2.10 / 2.11)$ driven by the Ubi promoter in snrk2.2/2.3 double mutant background, and several homozygous transgenic lines were obtained for each transgene. The Atsnrk2.2/ 2.3 double mutant exhibits ABA-insensitive phenotypes during seed germination and seeding growth [19]. To check whether the overexpression of $Z m S n R K 2$ genes affects the ABA sensitivity of the Atsnrk2.2/2.3 double mutant, the seeds from the WT, Atsnrk2.2/2.3 mutant and transgenic plants were germinated on the media supplemented with different concentrations of ABA. As shown in Additional file 1: Fig. S6, no difference in seeds germination was observed among the WT, Atsnrk2.2/2.3 mutant and the transgenic plants under normal conditions. In the presence of exogenous ABA, however, the seeds germination and seeding growth of $Z m S n R K 2.8$ and $Z m S n R K 2.10$ transgenic plants in Atsnrk2.2/2.3 mutant background was obviously inhibited compared to Atsnrk2.2/2.3 mutants, but less serious than the WT. In contrast, the $Z m S n R K 2.3$ and $Z m S n R K 2.11$ overexpressing plants in Atsnrk2.2/2.3 mutant background displayed a similar phenotype as Atsnrk2.2/2.3 mutant under ABA treatment. These results indicated that $Z m S n R K 2.8$ and $Z m S n R K 2.10$ possess a similar function as AtSnRK2.2 and AtSnRK2.3 in ABA signaling pathways. The germination rate of $Z m S n R K 2.10$ overexpression in Atsnrk2.2/ 2.3 mutant was further statistically analyzed. As Fig. 6 showed, at the 10th day after sown on $1 / 2 \mathrm{MS}$ agar medium containing $1.2 \mu \mathrm{M}$ ABA, approximately $50 \%$ seeds of the two OEZmSnRK2.10/Atsnrk2.2/2.3 lines comparing with $26 \%$ of the WT and $100 \%$ of Atsnrk2.2/ 2.3 mutant seeds germinated, which further confirmed that $Z m S n R K 2.10$ could partially complement the ABA insensitive phenotype of Atsnrk2.2/2.3.

We also analyzed the flowering time of the transgenic lines that expressed $Z m S n R K 2.10$ in the WT and Atsnrk2.2/2.3 mutant background (Fig. 7A-C). Comparing with the WT plants, the Atnrk2.2/2.3 double mutants showed an early flowering phenotype. Overexpression of $Z m S n R K 2.10$ in Atsnrk2.2/2.3 background, however, could restore the flowering time of the Atsnrk2.2/2.3 double mutants to WT level. ZmSnRK2.10 in WT background also resulted in a significant delay of flowering time. In the transgenic plants overexpressing ZmSnRK2.11 gene in both WT and Atsnrk2.2/2.3 mutant background, the flowering time was not affected. (Additional file 1: Fig. S7), suggesting that ZmSnRK2.10

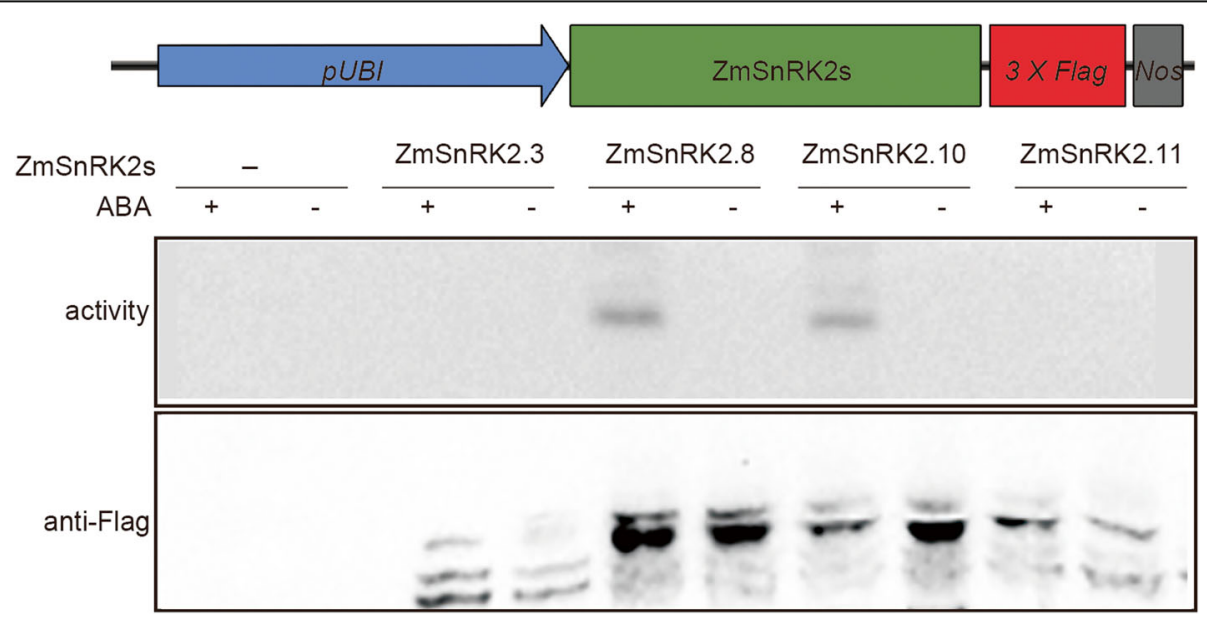

Fig. 5 The ZmSnRK2.10 kinase activity is activated by ABA. Mazie leaf protoplasts were transiently transformed with empty vector or the constructs expressing the indicated SnRK2s. The SnRK2 proteins were immunoprecipitated by using anti-Flag antibody (lower panel) and the kinase activity of SnRK2s was analyzed by in vitro kinase assay using MBP as a substrate (upper panel) 


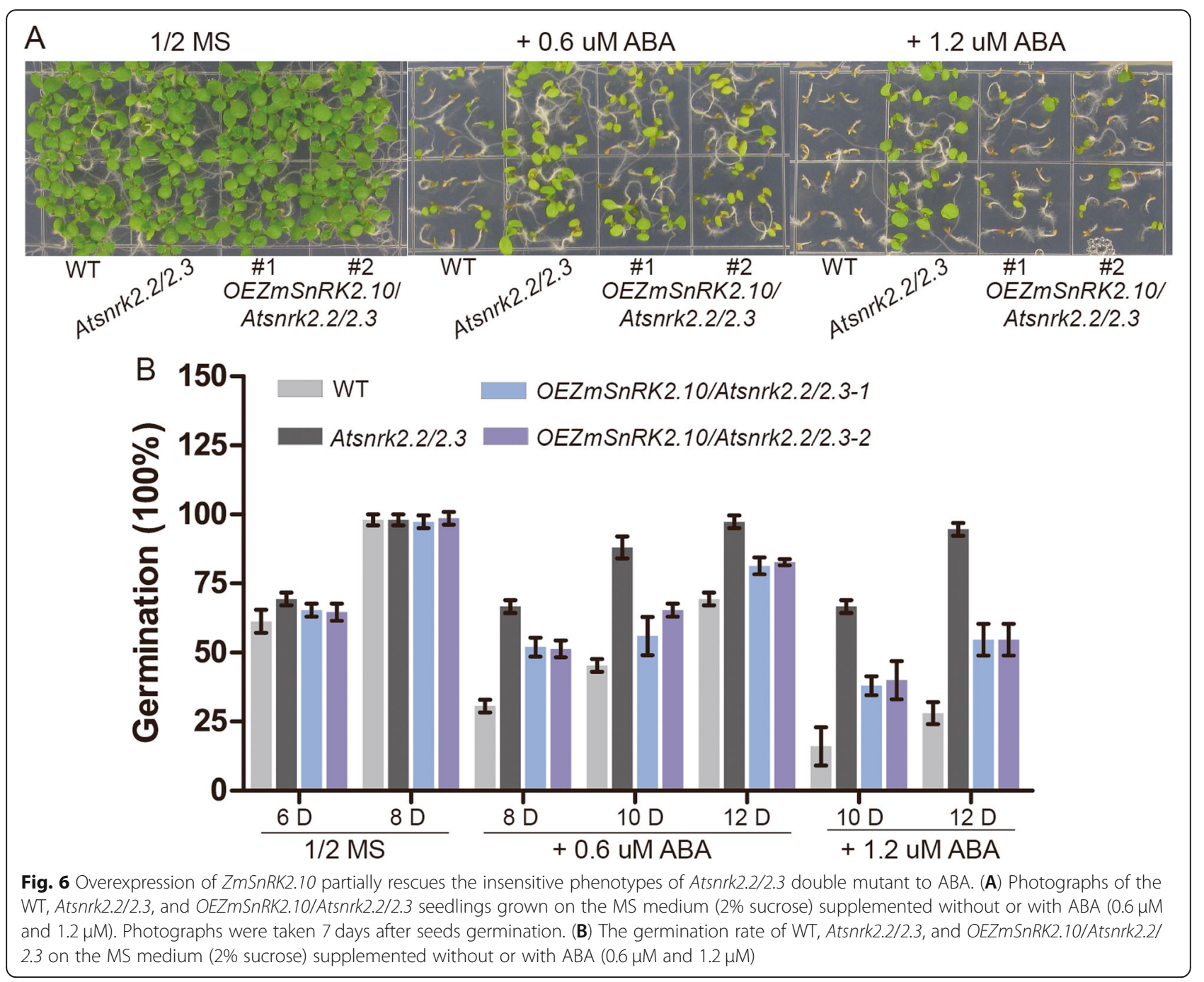

has a special role in controlling flowering time in Arabidopsis. To understand the mechanism of ZmSnRK2.10 in the regulation of flowering time, the transcript levels of two critical genes, FLOWERING LOCUS T (FT) and FLOWERING LOCUS C (FLC), in transgenic lines were analyzed by qRT-PCR. FT controls flowering time in Arabidopsis, and its mutant plants show significantly late flowering [33]. $F L C$ is a floral repressor and $F T$ can directly bind to the promoter region of $F L C$ to inhibit its expression, thus inhibiting the flowering in Arabidopsis [34]. The qRT-PCR analysis indicated that the expression level of $F L C$ was up-regulated and the expression level of $F T$ was reduced in the transgenic plants overexpressing ZmSnRK2.10 (Fig. 7D-E). Therefore, we suspected that overexpression of $\mathrm{ZmSnRK2.10}$ delayed flowering time at least partially via the regulation of the expression of FLC and FT genes.

In addition, nearly all major ABA responses are blocked in the Atsnrk2.2/2.3/2.6 triple mutant in
Arabidopsis. The triple mutant shows earlier flowering, smaller plant size, fewer pollen number, and poor fertilization phenotype compared with Atsnrk2.2/2.3 double mutant $[6,16]$. We also transformed ZmSnRK2.10 into Atsnrk2.2/3/6 triple mutant and obtained overexpression transgenic lines (Additional file 1: Fig. S8A, B). Overexpression of $Z m S n R K 2.10$ rescued the dwarf morphology of Atsnrk2.2/2.3/2.6 mutant significantly, but the plant size of transgenic plants was still smaller than the WT (Fig. 8 and Additional file 1: Fig. $\mathrm{S} 8 \mathrm{C})$. This result indicated that $\mathrm{ZmSnRK2.10}$ functions redundantly with AtSnRK2.2/2.3/2.6.

\section{Discussion}

The conserved evolution of the ZmSnRK2s

The core components of ABA signaling pathway have been well characterized in the dicot model plant Arabidopsis, but the studies on ABA regulatory pathway in crops still need to be strengthened. Protein kinase 

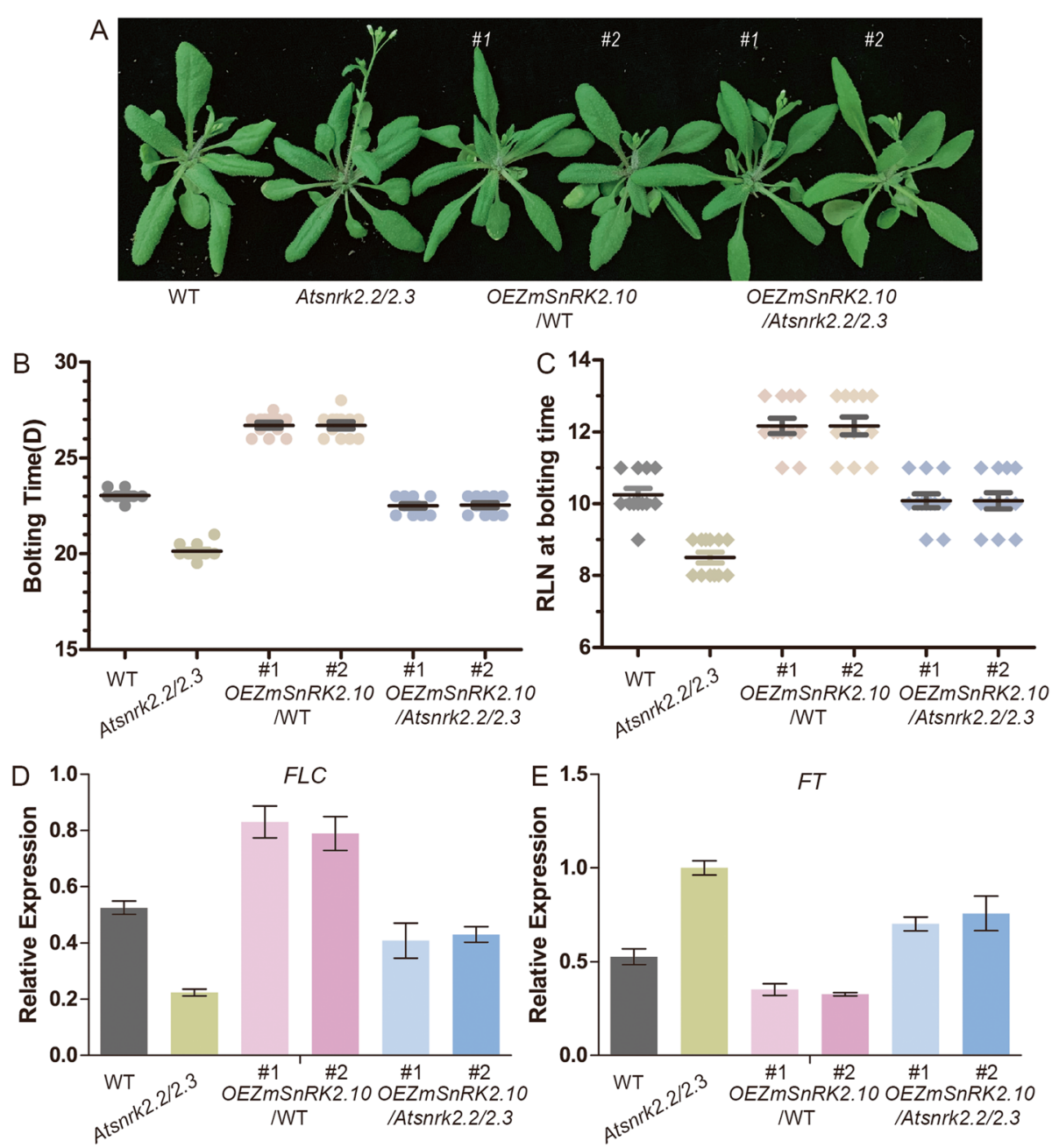

Fig. 7 Overexpression of ZmSnRK2.10 leads to delayed flowering time in Arabidopsis. (A) Flowering phenotype of the WT, Atsnrk2.2/2.3, OEZmSnRK2.10/WT, and OEZmSnRK2.10/Atsnrk2.2/2.3. The photograph was taken 35 days after growth under LD conditions. (B) Flowering time of the WT, Atsnrk2.2/2.3, OEZmSnRK2.10/WT, and OEZmSnRK2.10/Atsnrk2.2/2.3 under LD conditions was scored based on the days from germination to bolting. (C) Flowering time of the WT, Atsnrk2.2/2.3, OEZmSnRK2.10/WT, and OEZmSnRK2.10/Atsnrk2.2/2.3 under LD conditions was scored based on the number of rosette leaves at flowering stage. (D) qRT-PCR analysis of FLC gene in the WT, Atsnrk2.2/2.3, OEZmSnRK2.10/WT, and OEZmSnRK2.10/Atsnrk2.2/2.3. (E) qRT-PCR analysis of FT gene in the WT, Atsnrk2.2/2.3, OEZmSnRK2.10/WT, and OEZmSnRK2.10/Atsnrk2.2/2.3. Error bars represent SE from three biological replicates

SnRK2s is the central node of ABA signal transduction $[35,36]$. The SnRK2 genes family has been identified in many plants. In maize, 11 SnRK2 genes have been reported previously [26]. In this study, we identified 14 members of SnRK2 at genome-wide level using the latest maize genome database. Compared with 10 members in Arabidopsis and 10 members in rice, the SnRK2 members in maize were expanded to $14[9,10]$. The expansion of SnRK2 genes in maize was attributed to gene duplication events, which is one of the important strategies that plants used to adapt to the surrounding environment [37, 38]. It has been reported that three duplication events, including whole-genome duplication, genomic tandem duplication and segmental duplication in plants [39]. Similar to Arabidopsis and rice, the SnRK2 genes in maize were divided into three subclasses (Additional file 1: Fig. S1 and Fig. S2), and the exonintron organizations of SnRK2 genes among these three species exhibited a high similarity (Fig. 2A), which indicates that the gene structures of SnRK2s are evolutionarily conserved in higher plant. Further, the collinearity relationship of SnRK2s indicated that SnRK2 genes had been differentiated before the differentiation of maize, rice and Arabidopsis (Fig. 2C). The most ancient land plants, such as Bryophytes and Charophyceae algae, only contain SnRK2 genes clustered in subclass III, and the subclasses I and II branches were evolved as new systems to adapt to osmotic stress conditions after the 

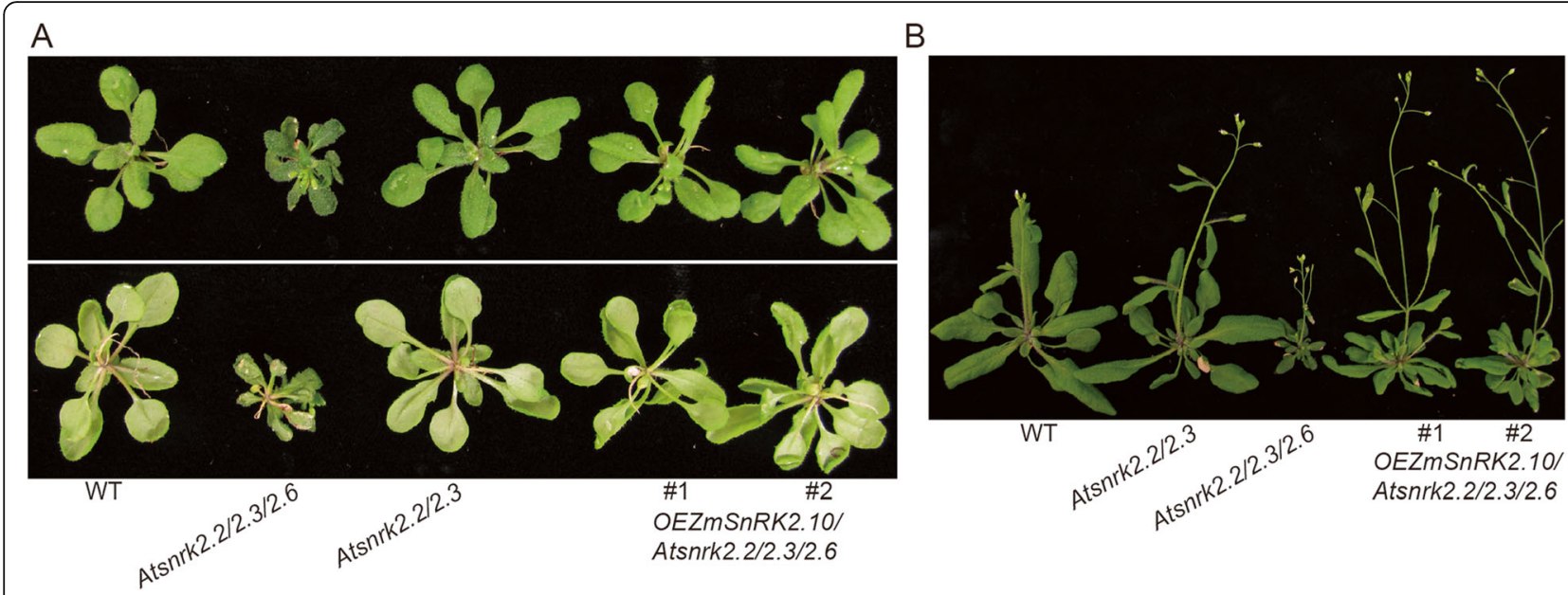

Fig. 8 Overexpression of ZmSnRK2.10 partially rescues the phenotype of Atsnrk2.2/2.3/2.6 in Arabidopsis. (A) The phenotype of the WT, Atsnrk2.2/ 2.3, Atsnrk2.2/2.3/2.6, and OEZmSnRK2.10/Atsnrk2.2/2.3/2.6. The photograph was taken 30 days after growth under LD conditions. (B) Flowering phenotype of the WT, Atsnrk2.2/2.3, Atsnrk2.2/2.3/2.6, and OEZmSnRK2.10/Atsnrk2.2/2.3/2.6. The photograph was taken 35 days after growth under LD condition

separation of bryophytes and vascular plants [38]. The neofunctionalization and subfunctionalization of the duplication gene is an important mechanism to maintain its stability. The original duplicated gene has dual functions and repulsion between these two functions, which results in gene loss. When the duplication event occurs, each gene obtains one of the functions and optimizes them respectively, leading to the elimination of functional conflict and the stability of duplicated genes [40]. This indicates that the duplication events of SnRK2 genes represent an ancient system and have expanded along with whole-genome duplication events in plants. Previous studies speculated that maize had experienced at least three genome-wide replication events: monocotyledonous plant differentiation, the emergence of Gramineae, and the differentiation of maize and sorghum. These duplication events occurred about 110 million years ago, 50 million years ago, and 12 million years ago, respectively [41-43]. In addition, through the synteny and collinearity analysis of SnRK2 genes in maize genome, we identified eight segmental duplications but no tandem duplication (Fig. 2B), which supports the opinion that most of maize genes were duplicated during ancient polyploidization [44]. These results suggested that the expansion of SnRK2 members and origin of novel gene functions in maize maybe due to the segment duplication and polyploidization. This explanation is consistent with the adaptive radiation model, which proposes that the newly developed genes help the existing genes to cope with new conditions, and the genomic tandem duplication accelerates the evolution of novel function of the duplicated genes $[45,46]$.
The expression pattern of $Z m S n R K 2 s$ in maize

Analysis of gene expression patterns can provide important clues to explore gene function. In Arabidopsis, the tissue-specific expression of SnRK2.6 in stomata is tightly coupled with its functions in guard cells to regulate stomatal closure [18]. The expression of two other subclass III genes, SnRK2.2 and SnRK2.3, are different from that of SnRK2.6. These two genes express in nearly all the tissues and are closely related to their role in regulating various ABA responses, such as seed germination, dormancy, and seedling growth $[19,28]$. Previous studies revealed the expression profile of $\mathrm{ZmSnRK2}$ family genes under different abiotic stresses [26, 31]. To investigate the spatiotemporal expression patterns of $Z m S n R K 2 s$, we first analyzed the expression of $Z m S n R K 2 s$ using public RNA-Seq database, and found that $Z m S n R K 2$ genes exhibited differential expression patterns during the growth and development of various maize tissues. Even in the same subclass, each gene exhibited a distinct expression pattern. The paralogous genes tend to maintain similar functions, but show differences in activity and specificity. The significant difference in gene expression among $Z m S n R K 2$ genes is consistent with the results reported in previous studies $[47,48]$. The changes of homologous genes in the promoter region lead to rapid divergence in expression patterns, which further lead to functional differentiation [49]. The chromosomal location, gene structure, and collinearity analysis indicated that four genes $Z m S n R K 2.8$, $Z m S n R K 2.9, Z m S n R K 2.10$, and ZmSnRK2.12, which belong to subclass III of SnRK2 in maize, are derived from gene duplication events. We focused on the differential expression pattern of these four genes and found that 
ZmSnRK2.10 was highly expressed in both embryo and endosperm, especially in the middle and late stage of kernel development, the stages of which are important for storage material accumulation and seed maturation (Fig. 3D). In addition, the transcripts of ZmSnRK2.10 were induced by ABA and sucrose respectively (Additional file 1: Fig. S3). In wheat, the transcripts of PKABA1 and TaSnRK2.3 were induced by ABA, and both genes are important for seed maturation and abiotic stress response $[8,27]$. Although the different maize tissues were tested in this research and previous reports $[26,31]$, the expression pattern of some $Z m S n R K 2 s$ under ABA treatment is almost consistent, such as $Z m S n R K 2.1$ and ZmSnRK2.7 (Additional file 1: Fig. S3). Moreover, the expression pattern of most $Z m S n R K 2 s$ in maize different tissues varied. For instance, the ZmSnRK2.10 showed an increased expression in kernel after ABA treatment, but was downregulated expression in seedling, leaf and root [26,31]. Combined with the results that $Z m S n R K 2.10$ was highly expressed in kernel and was induced by ABA, we propose that ZmSnRK2.10 plays important roles in linking metabolism and stress signaling during the grain filling and maturation process.

Functional characterization of ZmSnRK2.10 in Arabidopsis $S n R K 2 s$ are divided into three subclasses based on ABA activation in Arabidopsis. Subclass III, including SnRK2.2, SnRK2.3 and SnRK2.6, are strongly activated by ABA, and subclass II, including SnRK2.7 and SnRK2.8, are slightly activated by ABA. The subclass I SnRK2s cannot be activated by ABA, but can be strongly activated by hyperosmotic stress except the SnRK2.9 [7, 11]. Because the subclass III SnRK2s are ABAdependent, the snrk2.2 or 2.3 single mutant exhibits weak ABA-insensitive phenotypes and snrk2.2/2.3 double mutant shows strong ABA-insensitive phenotypes during seed germination and seedling growth in Arabidopsis [19]. Further studies showed that snrk2.2/ 2.3/2.6 triple mutant exhibits almost completely insensitive to ABA in seed germination and seedling growth $[16,28]$. SAPK8, SAPK9, and SAPK10, which are homologs of AtSnRK2.2, AtSnRK2.3, and AtSnRK2.6, belong to subclass III in rice and are strongly activated by ABA [10]. Overexpression of SAPK8, SAPK9, or SAPK10 in rice leads to delayed germination and seedling growth, which are caused by the accumulation of higher levels of ABA compared with WT plants [21]. Compared with SAPK8 and SAPK9, SAPK10 displays the highest homology to AtSnRK2.2 and AtSnRK2.3 and it mediates the phosphorylation of WRKY72, which results in the release of its suppression on jasmonic acid biosynthesis and bacterial blight resistance [50]. Overexpression of SAPK10 confers rice with hypersensitivity to ABA during seed germination and seedling growth [51]. In maize, we found that the $Z m S n R K 2.10$ was the closest homologous gene of SAPK10 in rice and $S n R K 2.2 / 2.3$ in Arabidopsis, and this gene, as well as $Z m S n R K 2.8$ (same as OST1 in Arabidopsis), was activated by ABA. The other subclass members $Z m S n R K 2.3$ and $Z m S n R K 2.11$ cannot be activated by ABA (Fig. 5 and Additional file 1: Fig. S5). Overexpression of $Z m S n R K 2.8$ rescues the droughthypersensitive phenotype of ost 1 mutant and significantly improves the growth and development of plants under stress conditions in Arabidopsis [32, 52] In our study, overexpression of $Z m S n R K 2.8$ and $Z m S n R K 2.10$, but not ZmSnRK2.3 and ZmSnRK2.11, in AtSnRK2.2/2.3 background can partially complement the hypersensitive phenotypes of Atsnrk2.2/2.3 to ABA in seed germination and early seedling development (Fig. 6 and Additional file 1: Fig. S6). In addition, overexpression of $Z m S n R K 2.10$ can also rescue the phenotype of Atsnrk2.2/2.3/2.6 triple mutant (Fig. 8 and Additional file 1: Fig. S8). The identified functions of $Z m S n R K .10$ in Arabidopsis suggests that the ABA-dependent members of $S n R K 2$ maybe are evolutionarily conserved in plants.

On the other hand, it has been reported that ABA can inhibit plant floral transition [53]. Exogenous application of ABA delays plant flowering time. A series of ABA synthesis and signal transduction mutants, including aba2, abi4, abi5, and snrk2.2/2.3/2.6, exhibit earlyflowering phenotype [6,53-55]. We found that the snrk2.2/2.3 double mutant also showed early flowering in Arabidopsis and overexpression of $\mathrm{ZmSnRK2.10}$ in snrk2.2/2.3 double mutant rescued early-flowering phenotype. Moreover, overexpression of $Z m S n R K 2.10$ in WT background delayed flowering time via the upregulation of FLC gene that is involved in floral transition (Fig. 7). This result is consistent with the interpretation that SnRK2-mediated phosphorylation of ABI5 and/or $\mathrm{ABF}$ promotes FLC gene expression [53]. All these results indicate that molecular functions of subclass III SnRK2s are conserved in plants. In future, overexpression or knock-out of $Z m S n R K 2.10$ by CRISPR-Cas9 technique needs to be performed in maize, which will advance our understanding of the roles of $\mathrm{ZmSnRK2.10}$ in the regulation of abiotic stress tolerance in maize.

\section{Conclusion}

Although $Z m S n R K 2 s$ have been cloned and characterized in maize as early as 2008, no genome-wide identification and characterization of $Z m S n R K 2 s$ has been conducted in maize. Here, we presented a genome-wide identification of $Z m S n R K 2 s$ in maize and found 14 $S n R K 2$ genes based on the released maize genome data. Through chromosomal localization, gene structure, evolutionary association, synteny and collinearity analysis of $S n R K 2$ genes in maize by comparing with the $S n R K 2 s$ in rice and Arabidopsis, we can learn that the $S n R K 2$ family 
genes are evolutionarily conserved in plants, especially the ABA-dependent subclass III SnRK2s. In addition, through transcriptional and biochemical analysis, we found that $Z m S n R K 2.10$ was highly expressed in kernel and was activated by ABA. Overexpression of ZmSnRK2.10 in snrk2.2/2.3 double and snrk2.2/2.3/2.6 triple mutants partially rescued the ABA-insensitive phenotypes of these mutants, and also resulted in delaying plant flowering in Arabidopsis. Our study provided new insights about the structure, evolution, and functions of SnRK2s in maize.

\section{Methods}

\section{Plant materials and growth condition}

Maize (Zea mays L.) self-interbreeding line B73 material (obtained from Maize Research Institute of Sichuan Agricultural University) used in this study, was grown on the experimental farm of Sichuan Agricultural University in Chongzhou, Sichuan, China. Seeds of Arabidopsis thaliana accession Col-0 (Columbia 0) and the mutants snrk2.2/2.3, snrk2.2/2.3/2.6 (all in Col-0 background, obtained from Zhu lab in Purdue University) [6, 19] were used in this study. The Arabidopsis plants, including transgenic lines, were grown under $16 \mathrm{~h}$ light $/ 8 \mathrm{~h}$ dark condition at $22^{\circ} \mathrm{C}$ in the day and $16^{\circ} \mathrm{C}$ at night.

\section{Whole genome identification of SnRK2s in Zea mays}

To identify the SnRK2 genes in Zea mays, we obtained the genome sequence and annotation data of Zea mays from MaizeGDB (https://www.maizegdb.org/). The protein database and genome database were blasted by using the query sequences of the SnRK2 family in Arabidopsis thaliana (http://www.arabidopsis.org) and Oryza sativa (http://www.ricedate.com). The E-value cut-off was set at 1.0e-5 to ensure confidence and redundant sequences were deleted by manual.

\section{Multiple sequence alignment, orthologous gene identification and phylogenetic analysis}

The phylogenetic analysis of SnRK2 family were conducted as the method in this article [56]. In brief, amino sequences from Zea mays, Arabidopsis thaliana and Oryza sativa were aligned by ClustalX v1.83 with default parameters and then constructed a phylogenetic tree using the neighbor joining (NJ) method in MEGA (version 5.10) [57]. The parameters of NJ analysis were as followed: bootstrap with 1000 replicates for statistical testing, pairwise deletion for data processing and poisson correction for modeling. The position in phylogenetic tree (bootstrap value $>50$ ) and identify between orthologous gene pairs $(>90 \%)$ would determine the orthologous gene in Zea mays, Arabidopsis thaliana and Oryza sativa.

\section{Chromosomal location of ZmSnRK2s and collinearity analysis}

Gene ID information of the ZmSnRK2 loci on chromosome were obtained from the genome annotation files. The distribution of $\mathrm{ZmSnRK2s}$ on the chromosome was generated with MapChart version 2.32 [58]. The Gene Structure Display Server (http://gsds.cbi.pku.edu.cn/) was used for gene structure analysis. Conserved protein motifs of the ZmSnRK2s were predicted by the MEME Version 5.0.4 program (http://meme-suite.org/). The default parameters of MEME were setting except two: maximum number of motifs, 25; optimum width, 6-50 [59].. The MCscanX program was used to identify ZmSnRK2s duplications as previous described [56, 60]. All genes were classified into various types of duplications, including WGD, segmental and tandem duplications. A schematic diagram of the putative WGD or segmental duplications of $\mathrm{ZmSnRK2s}$ was constructed using the Circos (http://circos.ca/), and the ZmSnRK2s with WGD or segmental duplications were linked by lines.

\section{Analysis of the expression pattern of ZmSnRK2 genes}

To study the expression patterns of the $Z m S n R K 2$ genes, we obtained the expression level of each $Z m S n R K 2$ gene in different tissues by analyzing the released transcription data [29], and then submitted it to the MeV_4_9_0 [61] for stratified clustering (Hierarchical Clustering, HCL). Further, different tissues of B73 were collected for RNA extract and qRT-PCR analysis, including root, stem, leaf, filament, anther at the same stage (Heading stage). Besides, the development kernels were also collected, including kernels at 2-30 days after pollination (DAP), endosperm and embryo at 15 DAP. The treatment to vitro kernel was according to the paper described by [30]. The RNA extraction of materials was followed the protocol of Trizol (TIANGEN, Beijing, China) reagent. RNA was reverse transcribed using the PrimeScript $^{\text {Tix }}$ RT Reagent Kit with genomic DNA Eraser (Takara, Dalian, China), and quantitative RT-PCRs were performed using a SYBR premix Ex Taqtm RT-PCR kit (Takara, Dalian, China). All the experiments were performed following the manufacturer's instructions. The primers used for qRT-PCR were listed in Additional file 1: Table S2.

\section{Gene cloning, vector construction and subcellular localization of ZmSnRK2 genes}

Full-length cDNA sequence of $Z m S n R K 2$ genes exclude terminator codon were amplified by PCR. The PCR products were sequenced and then sub-cloned into the pCAMBIA 2300-35S-eGFP vector [62]. Then the pCAMBIA2300-ZmSnRK2-eGFP fusion protein were transformed into Agrobacterium tumefaciens strain 
GV3101. The empty pCAMBIA2300-35S-eGFP was used as a control. The Agrobacterium was infected into $\mathrm{Ni}$ cotiana benthamiana leaves, and the fluorescence signal of green fluorescent protein (GFP) was observed by confocal microscope (Leica, Wetzlar, Germany). The ZmSnRK2.14 was not subcloned due to its low expression in almost all tissues. The primers used for vector contractor were listed in Additional file 1: Table S2.

\section{Isolation and transfection of protoplast}

The isolation of protoplasts in maize were performed as described with minor modification [63]. The middle parts $(7 \mathrm{~cm})$ of the second leaves of B73 were transversally cut into $0.5-0.8 \mathrm{~mm}$ long strips and put into a petri dish containing $0.6 \mathrm{M}$ mannitol for $5 \mathrm{~min}$. Then the strips were transferred into another dish containing enzyme solution [0.6 M mannitol, $20 \mathrm{mM}$ MES, $\mathrm{pH} 5.7$, $0.1 \%$ cellulase RS, $0.01 \%$ macerozyme, $0.1 \%$ BSA, $1 \mathrm{mM}$ $\mathrm{CaCl}_{2}$ and $\mathrm{ddH}_{2} \mathrm{O}$. After vacuum $(15-20 \mathrm{~cm}-\mathrm{Hg})$ distillation at room temperature for $20 \mathrm{~min}$, the dish was incubated at $28^{\circ} \mathrm{C}$ in the dark with shaking for $3 \mathrm{~h}$ at 40 $\mathrm{rpm}$ and for $10 \mathrm{~min}$ at $80 \mathrm{rpm}$. Then, the protoplasts were released. The enzyme solution containing protoplasts was transferred into sterile tubes by filtering with $35 \mu \mathrm{m}$ nylon mesh. The protoplasts were collected by centrifugation at $110 \times \mathrm{g}$ for $4 \mathrm{~min}$ at room temperature and washed once with $0.6 \mathrm{M}$ mannitol. Finally, the protoplasts were resuspended in $\mathrm{MMg}$ solution $(0.6 \mathrm{M}$ mannitol, $4 \mathrm{mM}$ MES, pH 5.7, $15 \mathrm{mM} \mathrm{MgC1}_{2}$ ) and observed under a light microscope to detect the quality and quantity.

The transfection of protoplast was performed with PEG-mediated protoplasts transformation protocol [63, 64]. The $20 \mu \mathrm{g}$ plasmid DNA of p2300-ZmSnRK2s were mixed with $200 \mu \mathrm{L}$ protoplasts (a concentration of $2 \times$ $10^{5}$ cells $\left./ \mathrm{mL}\right)$. About $220 \mu \mathrm{L}$ PEG solution $(0.8 \mathrm{M}$ mannitol, 40\% PEG 4000 and $100 \mathrm{mM} \mathrm{CaCl} 2$,] was immediately mixed with the protoplasts and plasmid DNA by gently shaking, and then incubated for $15 \mathrm{~min}$ at $25^{\circ} \mathrm{C}$. After incubation, the mixture of protoplasts was washed and collected by centrifugation, and then resuspended in incubation solution (4 mM MES, pH 5.7, 0.6 M mannitol, $4 \mathrm{mM} \mathrm{KCl}$ ) and incubated in the dark at $25^{\circ} \mathrm{C}$ overnight. The fluorescence signal was observed by the confocal microscope (Leica, Wetzlar, Germany).

Co-transfected ZmSnRK2.3, ZmSnRK2.8, ZmSnRK2.10 and $\mathrm{ZmSnRK2.11}$ with reporter gene to reconstitute the ABA signaling pathway in B73 leaves protoplast respectively. The ABA-responsive reporter and internal control were selected with RD29B-LUC and ZmUbi-GUS respectively. After transfection, the protoplasts were incubated in the absence/ presence of $50 \mu \mathrm{M} \mathrm{ABA}$ for $2 \mathrm{~h}$ under light for further analysis.

\section{Construction of transgenic plants}

The sequencing-confirmed ORFs of ZmSnRK2.3, $Z m S n R K 2.8, Z m S n R K 2.10$ and $Z m S n R K 2.11$ were cloned into the pCAMBIA3301 vector (CAMBIA, Canberra, Australia) respectively. The constructs were transferred into Agrobacterium tumefaciens strain GV3101 using electroporation method and then transformed into Arabidopsis using the floral tip method [65]. The seeds of the $\mathrm{T}_{0}$ generation were harvested and sown in soil, and the transformants $T_{1}$ plants were identified by spraying with Basta (glufosinate ammonium; Solarbio, Beijing, China) and confirmed by PCR to amplify 35S:ZmSnRK2s fragment and bar gene, respectively. The confirmed transgenic plants were harvested individually. The $\mathrm{T}_{2}$ seeds were placed on 1/2MS (Murashige \& Skoog) agar medium (1\%) containing $5 \mathrm{mg} / \mathrm{L}$ glufosinate ammonium. Seeds of $\mathrm{T}_{3}$ were collected from transgenic lines with a 3:1 (resistant: sensitive) segregation ratio. The two individual $\mathrm{T}_{3}$ lines displaying 100\% Basta resistance were considered homozygous and used for further experiments. The Arabidopsis Col-0 was used as control. All seeds of WT, mutants and transgenic plants were collected at the same stage.

\section{Germination assays and phenotype identification}

Seeds were sterilized for $20 \mathrm{~min}$ with $75 \%(\mathrm{v} / \mathrm{v})$ ethanol and $0.1 \%(\mathrm{v} / \mathrm{v})$ Tween 80 , then washed with ethanol twice and air-dry. Fifty seeds of each transgenic line, mutant and WT were placed on the 1/2MS agar medium (1.0\%) supplemented with different concentrations of ABA $(0.5,0.6,1.0,1.2$ or $1.5 \mu \mathrm{M})$, maintained at $4{ }^{\circ} \mathrm{C}$ in dark for 2 days, and then transferred to the growth chamber at $65 \%$ relative humidity under 16 -h -light at $22^{\circ} \mathrm{C}$ and 8 -h-dark at $18{ }^{\circ} \mathrm{C}$. The percentage of emergence of two green cotyledons was measured daily at 8 days after seeds were placed at room temperature.

The flowering time phenotype of WT, mutants and transgenic lines were compared by measuring the bolting time of each lines. Recording the numbers of leaf (RLN) would be another trait to measure the flowering time.

\section{GUS-staining assays}

In the GUS assay, GUS activity was performed following this paper [66] with few modifications. The cutting leaves were transferred into a GUS staining solution (1 mM X-Gluc, $10 \mathrm{mM}$ phosphate buffer $[\mathrm{pH}$ 7.0], $0.5 \%[\mathrm{v} / \mathrm{v}]$ Triton $\mathrm{X}-100$ and $2 \mathrm{mM}$ potassium ferricyanide). After vacuum infiltration and overnight shaking incubation at room temperature, the leaves were de-stained by repeated washes in $70 \%$ ethanol and photographed. 


\section{Protein extraction and immunoblot analysis}

For the experiment shown in Fig.5, Additional Files: Fig. S6, S7 and S8, protein extraction and immunoblot analysis were performed as described by [11] with minor modifications. The protein extraction buffer $(100 \mathrm{~mm}$ HEPES, pH 7.5, $5 \mathrm{~mm}$ EDTA, $5 \mathrm{~mm}$ EGTA, $2 \mathrm{~mm}$ orthovanadate, $10 \mathrm{~mm} \mathrm{NaF}, 20 \mathrm{~mm} \beta$-glycerophosphate, $5 \mathrm{~mm}$ DTT, protease inhibitor cocktail [Sigma-Aldrich] for plant cell and tissue extracts 1:100, and $50 \mu \mathrm{M}$ PMSF) was used to extracted the liquid nitrogen grounded leaves of transgenic plants. Samples were centrifuged at $4{ }^{\circ} \mathrm{C}$ and high speed for $10 \mathrm{~min}$, and supernatant was collected. Protein concentration was determined using BioRad protein assay. Immediately after isolation, about $15 \mu \mathrm{g}$ protein fractions were transferred to SDS loading buffer and separated by SDS-PAGE (12.5\% acrylamide). The western blotting detection protein was performed referring to this paper [30].

\section{Abbreviations}

ABA: abscisic acid: BSA: bovine serum albumin: CaMV: cauliflower mosaic virus; CDS: sequence coding for amino acids in protein; DAPI: 4', 6diamidino-2-phenylindole; EDTA: ethylene diamine tetraacetic acid; EGTA: ethylene glycol-bis(2-aminoethylether)-N, N, N', N'-tetraacetic acid; GUS: $\beta$-glucuronidase; HEPES: 2-[4-(2-hydroxyethyl) piperazin-1-yl] ethanesulfonic acid; MES: morpholinoethanesulfonic acid; MS: Murashige \& Skoog; ORF: open reading frame; PEG: polyethylene glycol;

PMSF: Phenylmethanesulfonyl fluoride; SDS-PAGE: sodium dodecyl sulfate polyacrylamide gel electrophoresis; Ubi: ubiquitin

\section{Supplementary Information}

The online version contains supplementary material available at https://doi. org/10.1186/s12870-021-03064-9.

\section{Additional file 1.}

\section{Acknowledgements}

We thank Dr. Jian-Kang Zhu (Shanghai Center for Plant Stress Biology and CAS Center for Excellence in Molecular Plant Sciences, Chinese Academy of Sciences) for providing the snrk2.2/2.3 and snrk2.2/2.3/2.6 mutants.

\section{Authors' contributions}

Y.B.H and Y.P.L conceived and supervised the research. Y.P.L and T.D.L designed the experiments. T.D.L, B.J.X, Y.Y.W, C.Q.M, Y.B.W performed the experiments. T.D.L and B.J.X wrote the original manuscript. Y.F.H, J.J.Z, H.M.L and G.W.Y analyzed part of the data. Y.H.L, H.H.H, C.Z.Z revised the manuscript. All authors have read and approved the manuscript.

\section{Funding}

This work was supported by the National Major Project for Transgenic Organism Breeding (2016ZX08003-001) and the Sichuan Science and Technology Program (2019YJ0428). The funding bodies played no role in study design, the collection and analysis of the data, data interpretation, and in writing the manuscript.

\section{Availability of data and materials}

The datasets supporting the conclusions of this study are available by contacting with the corresponding author (yubihuang@sohu.com). The RNASeq data are available in NCBI (www.ncbi.nlm.nih.gov) with the accession number SRP037559.

\section{Declarations}

Ethics approval and consent to participate

Not applicable.

Consent for publication

Not applicable.

\section{Competing interests}

The authors declare that they have no competing interests.

\section{Author details}

${ }^{1}$ State Key Laboratory of Crop Gene Exploration and Utilization in Southwest China, Chengdu 611130, Sichuan, China. ${ }^{2}$ College of Agronomy, Sichuan Agricultural University, No.211 Huimin Rd., Wenjiang Dist, Chengdu 611130, Sichuan, China. ${ }^{3}$ Triticeae Research Institute, Sichuan Agricultural University, Chengdu 611130, Sichuan, China. ${ }^{4}$ College of Life Science, Sichuan Agricultural University, Ya'an 625014, Sichuan, China. ${ }^{5}$ Maize Research Institute, Sichuan Agricultural University, Chengdu 611130, Sichuan, China. ${ }^{6}$ Shanghai Center for Plant Stress Biology and CAS Center for Excellence in Molecular Plant Sciences, Chinese Academy of Sciences, Shanghai 200032, China.

Received: 25 September 2020 Accepted: 25 May 2021

Published online: 01 July 2021

\section{References}

1. Chen K, Li G-J, Bressan RA, Song CP, Zhu J-K, Zhao Y. Abscisic acid dynamics, signaling, and functions in plants. J Integr Plant Biol. 2020;62(1): 25-54. https://doi.org/10.1111/jipb.12899.

2. Zhang D-P. Abscisic acid: metabolism, transport and signaling: Springer; 2014

3. Ma Y, Szostkiewicz I, Korte A, Moes D, Yang Y, Christmann A, et al. Regulators of PP2C phosphatase activity function as abscisic acid sensors. Science. 2009;324(5930):1064-8. https://doi.org/10.1126/science.1172408.

4. Park S-Y, Fung $P$, Nishimura N, Jensen DR, Fujii $H$, Zhao $Y$, et al. Abscisic acid inhibits type $2 \mathrm{C}$ protein phosphatases via the PYR/PYL family of START proteins. Science. 2009;324(5930):1068-71. https://doi.org/10.1126/science.11 73041.

5. Fujii H, Chinnusamy V, Rodrigues A, Rubio S, Antoni R, Park S-Y, et al. In vitro reconstitution of an abscisic acid signalling pathway. Nature. 2009;462(7273): 660-4. https://doi.org/10.1038/nature08599.

6. Wang P, Xue L, Batelli G, Lee S, Hou Y-J, Van Oosten MJ, et al. Quantitative phosphoproteomics identifies SnRK2 protein kinase substrates and reveals the effectors of abscisic acid action. P Natl Acad Sci USA. 2013;110(27): 11205-10. https://doi.org/10.1073/pnas.1308974110.

7. Lin Z, Li Y, Zhang Z, Liu X, Hsu C-C, Du Y, Sang T, Zhu C, Wang Y, Satheesh $V$, et al. A RAF-SnRK2 kinase cascade mediates early osmotic stress signaling in higher plants. Nat Commun 2020;11(1):1-10.

8. Anderberg RJ, Walker-Simmons MK. Isolation of a wheat cDNA clone for an abscisic acid-inducible transcript with homology to protein kinases. P Natl Acad Sci USA. 1992;89(21):10183-7. https://doi.org/10.1073/pnas.89.21.10183.

9. Hrabak EM, Chan CWM, Gribskov M, Harper JF, Choi JH, Halford N, et al. The Arabidopsis CDPK-SnRK superfamily of protein kinases. Plant Physiol. 2003; 132(2):666-80. https://doi.org/10.1104/pp.102.011999.

10. Kobayashi Y, Yamamoto S, Minami H, Kagaya Y, Hattori T. Differential activation of the rice sucrose nonfermenting1-related protein kinase2 family by hyperosmotic stress and abscisic acid. Plant Cell. 2004;16(5):1163-77. https://doi.org/10.1105/tpc.019943.

11. Boudsocq $M$, Barbier-Brygoo $H$, Lauriere $C$. Identification of nine sucrose nonfermenting 1-related protein kinases 2 activated by hyperosmotic and saline stresses in Arabidopsis thaliana. J biol Chem. 2004:279(40):41758-66.

12. Mizoguchi M, Umezawa T, Nakashima K, Kidokoro S, Takasaki H, Fujita Y, et al. two closely related subclass II SnRK2 protein kinases cooperatively regulate drought-inducible gene expression. Plant Cell Physiol. 2010;51(5): $842-7$.

13. Umezawa T, Nakashima K, Miyakawa T, Kuromori T, Tanokura M, Shinozaki K, et al. Molecular basis of the Core regulatory network in ABA responses: sensing. Signaling and Transport Plant Cell Physiol. 2010;51(11):1821-39. https://doi.org/10.1093/pcp/pcq156. 
14. Yang G, Yu Z, Gao L, Zheng C. SnRK2s at the crossroads of growth and stress responses. Trends Plant Sci. 2019;24(8):672-6. https://doi.org/10.1016/j. tplants.2019.05.010.

15. Boudsocq M, Droillard M-J, Barbier-Brygoo H, Lauriere C. Different phosphorylation mechanisms are involved in the activation of sucrose nonfermenting 1 related protein kinases 2 by osmotic stresses and abscisic acid. Plant Mol Biol. 2007;63(4):491-503. https://doi.org/10.1007/s11103-006-91 03-1.

16. Fujii $\mathrm{H}$, Verslues PE, Zhu J-K. Arabidopsis decuple mutant reveals the importance of SnRK2 kinases in osmotic stress responses in vivo. P Natl Acad Sci USA. 2011;108(4):1717-22. https://doi.org/10.1073/pnas.1018367108.

17. Yoshida R, Hobo T, Ichimura K, Mizoguchi T, Takahashi F, Aronso J, et al. ABA-activated SnRK2 protein kinase is required for dehydration stress signaling in Arabidopsis. Plant Cell Physiol. 2002;43(12):1473-83. https://doi. org/10.1093/pcp/pcf188.

18. Mustilli AC, Merlot S, Vavasseur A, Fenzi F, Giraudat J. Arabidopsis OST1 protein kinase mediates the regulation of stomatal aperture by abscisic acid and acts upstream of reactive oxygen species production. Plant Cell. 2002; 14(12):3089-99. https://doi.org/10.1105/tpc.007906.

19. Fujii $H$, Verslues $P E$, Zhu J-K. Identification of two protein kinases required for abscisic acid regulation of seed germination, root growth, and gene expression in Arabidopsis. Plant Cell. 2007;19(2):485-94. https://doi.org/10.11 05/tpc.106.048538

20. Saha J, Chatterjee C, Sengupta A, Gupta K, Gupta B. Genome-wide analysis and evolutionary study of sucrose non-fermenting 1-related protein kinase 2 (SnRK2) gene family members in Arabidopsis thaliana and Oryza sativa. Comput Biol Chem. 2014;49:59-70. https://doi.org/10.1016/j. compbiolchem.2013.09.005.

21. Lin Q, Wu F, Sheng P, Zhang Z, Zhang X, Guo X, et al. The SnRK2-APC/C-TE regulatory module mediates the antagonistic action of gibberellic acid and abscisic acid pathways. Nat Commun. 2015;6(1):1-10.

22. McCarty DR, Hattori T, Carson CB, Vasil V, Lazar M, Vasil IK. The Viviparous-1 developmental gene of maize encodes a novel transcriptional activator. Cell. 1991;66(5):895-905. https://doi.org/10.1016/0092-8674(91)90436-3.

23. McCarty DR. Genetic control and integration of maturation and germination pathways in seed development. Annu Rev Plant Biol. 1995;46(1):71-93. https://doi.org/10.1146/annurev.pp.46.060195.000443.

24. Young TE, Gallie DR. Programmed cell death during endosperm development. Plant Mol Biol. 2000;44(3):283-301. https://doi.org/10.1023/A:1 026588408152.

25. Young TE, Gallie DR. Regulation of programmed cell death in maize endosperm by abscisic acid. Plant Mol Biol. 2000;42(2):397-414. https://doi. org/10.1023/A:1006333103342.

26. Huai J, Wang M, He J, Zheng J, Dong Z, Lv H, et al. Cloning and characterization of the SnRK2 gene family from Zea mays. Plant Cell Rep. 2008;27(12):1861-8. https://doi.org/10.1007/s00299-008-0608-8.

27. Tian S, Mao X, Zhang H, Chen S, Zhai C, Yang S, et al. Cloning and characterization of TaSnRK2.3, a novel SnRK2 gene in common wheat. J Exp Bot. 2013;64(7):2063-80. https://doi.org/10.1093/jxb/ert072.

28. Nakashima K, Fujita Y, Kanamori N, Katagiri T, Umezawa T, Kidokoro S, et al. Three Arabidopsis SnRK2 protein kinases, SRK2D/SnRK2.2, SRK2E/SnRK2.6/ OST1 and SRK2I/SnRK2.3, involved in ABA signaling are essential for the control of seed development and dormancy. Plant Cell Physiol. 2009;50(7): 1345-63. https://doi.org/10.1093/pcp/pcp083.

29. Chen J, Zeng B, Zhang M, Xie S, Wang G, Hauck A, et al. Dynamic transcriptome landscape of maize embryo and endosperm development. Plant Physiol. 2014;166(1):252-64. https://doi.org/10.1104/pp.114.240689.

30. Li Y, Yu G, LV Y, Long T, Li P, Hu Y, et al. Combinatorial interaction of two adjacent cis-active promoter regions mediates the synergistic induction of Bt2 gene by sucrose and ABA in maize endosperm. Plant Sci. 2018;274:33240. https://doi.org/10.1016/j.plantsci.2018.06.003.

31. Wang $Y G$, Fu FL, Yu HQ, Hu T, Zhang YY, Tao $Y$, et al. Interaction network of core ABA signaling components in maize. Plant Mol Biol. 2018;96(3):245-63. https://doi.org/10.1007/s11103-017-0692-7.

32. Vilela B, Moreno-Cortés A, Rabissi A, Leung J, Pagès M, Lumbreras V. The maize OST1 kinase homolog phosphorylates and regulates the maize SNAC1-type transcription factor. PLoS One. 2013;8(2):e58105. https://doi. org/10.1371/journal.pone.0058105.

33. Koornneef $M$, Hanhart $C J$, Veen $J H$. A genetic and physiological analysis of late flowering mutants in Arabidopsis thaliana. Mol Gen Genet. 1991;229(1): 57-66. https://doi.org/10.1007/BF00264213.
34. Bastow R, Mylne JS, Lister C, Lippman Z, Martienssen RA, Dean C. Vernalization requires epigenetic silencing of FLC by histone methylation. Nature. 2004;427(6970):164-7. https://doi.org/10.1038/na ture02269.

35. Cutler SR, Rodriguez PL, Finkelstein RR, Abrams SR. Abscisic acid: emergence of a Core signaling network. Annu Rev Plant Biol. 2010;61(1):651-79. https:// doi.org/10.1146/annurev-arplant-042809-112122.

36. Kulik A, Wawer I, Krzywinska E, Bucholc M, Dobrowolska G. SnRK2 protein kinases-key regulators of plant response to abiotic stresses. Omics. 2011; 15(12):859-72. https://doi.org/10.1089/omi.2011.0091.

37. Cronk QCB. Plant evolution and development in a post-genomic context. Nat Rev Genet. 2001;2(8):607-19. https://doi.org/10.1038/35084556.

38. Shinozawa A, Otake R, Takezawa D, Umezawa T, Komatsu K, Tanaka K, et al. SnRK2 protein kinases represent an ancient system in plants for adaptation to a terrestrial environment. Commun Biol. 2019;2(1):1-13.

39. Zhang JZ. Evolution by gene duplication: an update. Trends Ecol Evol. 2003; 18(6):292-8. https://doi.org/10.1016/S0169-5347(03)00033-8.

40. Hughes AL. The evolution of functionally novel proteins after gene duplication. P Roy Soc B-Biol Sci. 1994;256(1346):119-24.

41. Lyons E, Freeling M. How to usefully compare homologous plant genes and chromosomes as DNA sequences. Plant J. 2008;53(4):661-73. https://doi. org/10.1111/j.1365-313X.2007.03326.x

42. Tang $H$, Bowers JE, Wang $X$, Ming $R$, Alam M, Paterson AH. Perspective Synteny and collinearity in plant genomes. Science. 2008;320(5875):486-8. https://doi.org/10.1126/science.1153917.

43. Lee $T-H$, Tang $H$, Wang $X$, Paterson AH. PGDD: a database of gene and genome duplication in plants. Nucleic Acids Res. 2013:41(D1):D1152-8.

44. Ahn S, Tanksley SD. Comparative linkage maps of the rice and maize genomes. P Natl Acad Sci USA. 1993;90(17):7980-4. https://doi.org/10.1073/ pnas.90.17.7980.

45. Zhao W, Cheng Y-H, Zhang C, Shen X-J, You Q-B, Guo W, et al. Genomewide identification and characterization of the GmSnRK2 family in soybean. Int J Mol Sci. 2017;18(9):1834. https://doi.org/10.3390/ijms18091834.

46. Francino MP. An adaptive radiation model for the origin of new gene functions. Nat Genet. 2005;37(6):573-7. https://doi.org/10.1038/ng1579.

47. Schnable JC, Springer NM, Freeling M. Differentiation of the maize subgenomes by genome dominance and both ancient and ongoing gene loss. P Natl Acad Sci USA. 2011;108(10):4069-74. https://doi.org/10.1073/pna s.1101368108.

48. Garcia N, Zhang W, Wu Y, Messing J. Evolution of gene expression after gene amplification. Genome Biol Evol. 2015;7(5):1303-12. https://doi.org/1 0.1093/gbe/evv075

49. Kaessmann $H$, Vinckenbosch $N$, Long M. RNA-based gene duplication: mechanistic and evolutionary insights. Nat Rev Genet. 2009;10(1):19-31. https://doi.org/10.1038/nrg2487.

50. Hou Y, Wang Y, Tang L, Tong X, Wang L, Liu L, et al. SAPK10-mediated phosphorylation on WRKY72 releases its suppression on jasmonic acid biosynthesis and bacterial blight resistance. Iscience. 2019;16:499-510. https://doi.org/10.1016/j.isci.2019.06.009.

51. Wang Y, Hou Y, Qiu J, Wang H, Wang S, Tang L, et al. Abscisic acid promotes jasmonic acid biosynthesis via a 'SAPK10-bZIP72-AOC' pathway to synergistically inhibit seed germination in rice (Oryza sativa). New Phytol. 2020:228(4):1336-53. https://doi.org/10.1111/nph.16774.

52. Ying S, Zhang D-F, Li H-Y, Liu Y-H, Shi Y-S, Song Y-C, et al. Cloning and characterization of a maize SnRK2 protein kinase gene confers enhanced salt tolerance in transgenic Arabidopsis. Plant Cell Rep. 2011;30(9):1683-99. https://doi.org/10.1007/s00299-011-1077-z.

53. Wang $Y$, Li L, Ye T, Lu Y, Chen X, Wu Y. The inhibitory effect of ABA on floral transition is mediated by ABI5 in Arabidopsis. J Exp Bot. 2013;64(2):675-84. https://doi.org/10.1093/jxb/ers361.

54. Domagalska MA, Sarnowska E, Nagy F, Davis SJ. Genetic analyses of interactions among gibberellin, abscisic acid, and Brassinosteroids in the control of flowering time in Arabidopsis thaliana. PLoS One. 2010;5(11): e14012. https://doi.org/10.1371/journal.pone.0014012.

55. Shu K, Chen Q, Wu Y, Liu R, Zhang H, Wang S, et al. ABSCISIC ACID-INSENS ITIVE 4 negatively regulates flowering through directly promoting Arabidopsis FLOWERING LOCUS C transcription. J Exp Bot. 2016;67(1):195205. https://doi.org/10.1093/jxb/erv459.

56. Zhao L, Youjun Lü, Chen W, et al. Genome-wide identification and analyses of the AHL gene family in cotton (Gossypium). BMC Genomics, 2020; 21(1):69. 
57. Hall BG. Building phylogenetic trees from molecular data with MEGA. Mol Biol Evol. 2013;30(5):1229-35. https://doi.org/10.1093/molbev/mst012.

58. Voorrips RE. MapChart: software for the graphical presentation of linkage maps and QTLs. J Hered. 2002;93(1):77-8. https://doi.org/10.1093//hered/ 93.1.77.

59. Hu B, Jin J, Guo A-Y, Zhang H, Luo J, Gao G. GSDS 2.0: an upgraded gene feature visualization server. Bioinformatics. 2015;31(8):1296-7. https://doi. org/10.1093/bioinformatics/btu817.

60. Wang Y, Tang H, DeBarry JD, Tan X, Li J, Wang X. Lee T-h, Jin H, Marler B, Guo H, et al. MCScanX: a toolkit for detection and evolutionary analysis of gene synteny and collinearity. Nucleic Acids Res. 2012;40(7):e49. https://doi. org/10.1093/nar/gkr1293.

61. Chu VT, Gottardo R, Raftery AE, Bumgarner RE, Yeung KY. MeV+R: using MeV as a graphical user interface for Bioconductor applications in microarray analysis. Genome Biol. 2008;9(7):R118. https://doi.org/10.1186/gb-2008-9-7r118.

62. Xiao Q, Wang Y, Du J, Li H, Wei B, Wang Y, et al. ZmMYB14 is an important transcription factor involved in the regulation of the activity of the $\mathrm{ZmBT1}$ promoter in starch biosynthesis in maize. FEBS J. 2017;284(18):3079-99. https://doi.org/10.1111/febs.14179.

63. Cao J, Yao D, Lin F, Jiang M. PEG-mediated transient gene expression and silencing system in maize mesophyll protoplasts: a valuable tool for signal transduction study in maize. Acta Physiol Plant. 2014;36(5):1271-81. https:// doi.org/10.1007/s11738-014-1508-x.

64. Yoo S-D, Cho Y-H, Sheen J. Arabidopsis mesophyll protoplasts: a versatile cell system for transient gene expression analysis. Nat Protoc. 2007;2(7): 1565-72. https://doi.org/10.1038/nprot.2007.199.

65. Clough SJ, Bent AF. Floral dip: a simplified method for Agrobacteriummediated transformation of Arabidopsis thaliana. Plant J. 1998;16(6):735-43. https://doi.org/10.1046/j.1365-313x.1998.00343.x.

66. Stangeland B, Salehian Z. An improved clearing method for GUS assay in Arabidopsis endosperm and seeds. Plant Mol Biol Rep. 2002;20(2):107-14. https://doi.org/10.1007/BF02799426.

\section{Publisher's Note}

Springer Nature remains neutral with regard to jurisdictional claims in published maps and institutional affiliations.

Ready to submit your research? Choose BMC and benefit from:

- fast, convenient online submission

- thorough peer review by experienced researchers in your field

- rapid publication on acceptance

- support for research data, including large and complex data types

- gold Open Access which fosters wider collaboration and increased citations

- maximum visibility for your research: over $100 \mathrm{M}$ website views per year

At $\mathrm{BMC}$, research is always in progress.

Learn more biomedcentral.com/submissions 